\title{
Supplementary Information for: Shallow-crustal metamorphism during Late Cretaceous anatexis in the Sevier hinterland plateau: peak temperature conditions from the Grant Range, eastern Nevada, U.S.A.
}

\author{
Sean P. Long ${ }^{1 *}$, and Emmanuel Soignard ${ }^{2}$ \\ ${ }^{1}$ School of the Environment, Washington State University, Pullman, WA 99164, U.S.A. \\ ${ }^{2}$ LeRoy Eyring Center for Solid State Science, Arizona State University, Tempe, AZ 85287, \\ U.S.A. \\ *address correspondence to: sean.p.long@wsu.edu, 509-335-8868
}

Discussion DR1: Supporting data for Raman spectroscopy on carbonaceous material (RSCM) thermometry

Multiple grains of carbonaceous material (CM) were analyzed from each sample. The center positions, heights, widths, and areas of four first-order Raman peaks, including the G peak and three defect bands, D1, D2, and D3, are shown for individual analyses on Table DR1. These parameters were determined using a peak fitting program written in Matlab by E. Soignard, which allowed peak shapes to be fit by a combination of gaussian and lorentzian peaks, and background slope to be removed by using a 1st-order polynomial. R1 and R2 correspond to the height and area ratios as defined in equations 1 and 2 of Rahl et al. (2005), and the peak temperature ( $T_{\text {peak }}$ ) for each analysis is calculated from equation 3 of Rahl et al. (2005). Analyses of each sample on Table DR1 are ordered from low to high peak temperature. Standard means of $\mathrm{R} 1, \mathrm{R} 2$, and $\mathrm{T}_{\text {peak }}$ for all analyses from each sample are shown. The internal variation of $\mathrm{R} 1, \mathrm{R} 2$, and $\mathrm{T}_{\text {peak }}$ from each sample is represented by 1 standard deviation on the mean. However, the calibration equation of Rahl et al. (2005) also introduces an external $\pm 50^{\circ} \mathrm{C}$ uncertainty in $T_{\text {peak }}$. Therefore, after Cooper et al. (2013), in order to present a more representative uncertainty, we calculated a propagated standard error (SE) by adding internal and external uncertainties quadratically, and dividing by the square root of the number of analyses (n). Mean $\mathrm{T}_{\text {peak }}$ with this propagated $2 \mathrm{SE}$ uncertainty is reported for each sample on Table 1 in the text. 
Table DR1: Supporting data for RSCM peak temperature determinations.

\begin{tabular}{|c|c|c|c|c|c|c|c|c|c|c|c|c|c|c|c|c|c|c|c|}
\hline \multirow{2}{*}{$\begin{array}{l}\text { sample and } \\
\text { spot number }\end{array}$} & \multicolumn{4}{|c|}{ peak center position } & \multicolumn{4}{|c|}{ peak width } & \multicolumn{4}{|c|}{ peak amplitude } & \multicolumn{4}{|c|}{ peak area } & \multicolumn{2}{|c|}{ ratios } & \multirow{2}{*}{$\begin{array}{l}\mathrm{T}_{\text {peak }} \\
\left({ }^{\circ} \mathrm{C}\right) \\
\end{array}$} \\
\hline & D1 & D3 & G & D2 & D1 & D3 & G & D2 & D1 & D3 & G & D2 & D1 & D3 & G & D2 & R1 & $\mathbf{R 2}$ & \\
\hline GR29_spot18 & 1350 & 1550 & 1606 & 1623 & 92 & 70 & 52 & 15 & 2020 & 50 & 1720 & 10 & 91917 & 3722 & 95117 & 160 & 1.174 & 0.754 & 198 \\
\hline GR29_spot12 & 1346 & 1570 & 1609 & 1623 & 65 & 80 & 44 & 15 & 4860 & 1050 & 3400 & 10 & 496215 & 89332 & 159095 & 160 & 1.429 & 0.757 & 223 \\
\hline GR29_spot9 & 1346 & 1570 & 1609 & 1623 & 63 & 90 & 43 & 15 & 1600 & 250 & 1130 & 10 & 158336 & 23928 & 51674 & 160 & 1.416 & 0.754 & 225 \\
\hline GR29_spot19 & 1344 & 1565 & 1607 & 1623 & 62 & 75 & 52 & 15 & 1710 & 200 & 910 & 10 & 166536 & 15952 & 50324 & 160 & 1.879 & 0.768 & 236 \\
\hline GR29_spot20 & 1345 & 1565 & 1607 & 1623 & 63 & 75 & 50 & 15 & 3030 & 250 & 1830 & 10 & 299849 & 19940 & 97308 & 160 & 1.656 & 0.755 & 242 \\
\hline GR29_spot13 & 1346 & 1565 & 1609 & 1623 & 66 & 80 & 46 & 15 & 1210 & 200 & 930 & 10 & 121392 & 17016 & 45495 & 160 & 1.301 & 0.727 & 243 \\
\hline GR29_spot10 & 1346 & 1569 & 1608 & 1623 & 58 & 80 & 44 & 15 & 2000 & 300 & 1210 & 10 & 170442 & 25523 & 56619 & 160 & 1.653 & 0.751 & 246 \\
\hline GR29_spot8 & 1345 & 1570 & 1606 & 1623 & 62 & 70 & 53 & 15 & 2250 & 130 & 1600 & 10 & 212049 & 9678 & 90182 & 160 & 1.406 & 0.702 & 280 \\
\hline GR29_spot11 & 1348 & 1575 & 1611 & 1623 & 57 & 70 & 44 & 15 & 1780 & 600 & 1100 & 10 & 128489 & 44666 & 51472 & 160 & 1.618 & 0.714 & 284 \\
\hline GR29_spot16 & 1345 & 1555 & 1610 & 1623 & 74 & 90 & 52 & 15 & 3330 & 570 & 2670 & 10 & 387076 & 54556 & 189915 & 160 & 1.247 & 0.671 & 296 \\
\hline GR29_spot7 & 1346 & 1570 & 1601 & 1623 & 54 & 70 & 62 & 15 & 1340 & 50 & 750 & 10 & 102650 & 3722 & 49451 & 160 & 1.787 & 0.675 & 333 \\
\hline \multirow[t]{5}{*}{ GR29_spot15 } & 1347 & 1565 & 1612 & 1623 & 74 & 70 & 55 & 15 & 1650 & 350 & 1440 & 10 & 173211 & 26055 & 108335 & 160 & 1.146 & 0.615 & 343 \\
\hline & & & & & & & & & & & & & & & & mean: & 1.466 & 0.721 & 261 \\
\hline & & & & & & & & & & & & & & & $1 \mathrm{SE}$ (i & ternal):| & 0.062 & 0.012 & 12 \\
\hline & & & & & & & & & & & & & & & prop. $2 \mathrm{~S}$ & linterna & l and ex & ternal): & 36 \\
\hline & & & & & & & & & & & & & & & & & & $\mathrm{n}:$ & 13 \\
\hline GR34_spot18 & 1357 & 1550 & 1583 & 1623 & 68 & 70 & 32 & 35 & 00 & 150 & 1 & 180 & 3 & 111 & 6350 & 6700 & 0.682 & 0.568 & 313 \\
\hline GR34_spot17 & 1358 & 1550 & 1584 & 1625 & 77 & 75 & 37 & 40 & 1050 & 100 & 2010 & 270 & 126999 & 7976 & 116820 & 11486 & 0.522 & 0.497 & 353 \\
\hline GR34_spot7 & 1353 & 1560 & 1585 & 1624 & 59 & 75 & 24 & 15 & 315 & 50 & 1190 & 60 & 29193 & 3988 & 44862 & 957 & 0.265 & 0.389 & 402 \\
\hline GR34_spot8 & 1358 & 1555 & 1584 & 1621 & 58 & 70 & 23 & 18 & 205 & 70 & & 50 & & & & 957 & 0.200 & 30 & 446 \\
\hline GR34_spot20 & 1363 & 1550 & 1585 & 1622 & 64 & 75 & 26 & 35 & 230 & 100 & 1000 & 90 & 21629 & 7976 & 40841 & 3350 & 0.230 & 0.329 & 456 \\
\hline GR34_spot19 & 1362 & 1550 & 1585 & 1623 & 63 & 70 & 23 & 40 & 200 & 80 & 1110 & 70 & 19792 & 5955 & 40102 & 2978 & 0.180 & 0.315 & 456 \\
\hline GR34_spot21 & 1360 & 1545 & 1585 & 1621 & 61 & 75 & 22 & 30 & 230 & 80 & & 110 & & & & 3509 & 0.181 & & 469 \\
\hline GR34_spot11 & 1358 & 1550 & 1585 & 1624 & 55 & 75 & 25 & 35 & 240 & 140 & 1000 & 130 & 18056 & 11166 & 39270 & 4839 & 0.240 & 0.315 & 474 \\
\hline GR34_spot14 & 1356 & 1550 & 1584 & 1622 & 52 & 75 & 25 & 15 & 310 & 10 & 2350 & 30 & 22868 & 798 & 92284 & 479 & 0.207 & 0.280 & 502 \\
\hline GR34_spot10 & 1360 & 1555 & 1584 & 1624 & 60 & 70 & 23 & 33 & 210 & 130 & & 150 & & 9678 & & & 0.152 & & 523 \\
\hline GR34_spot9 & 1359 & 1550 & 1584 & 1623 & 61 & 70 & 22 & 20 & 180 & 70 & 1540 & 70 & 15576 & 5211 & 53219 & 1489 & 0.117 & 0.226 & 533 \\
\hline GR34_spot13 & 1359 & 1550 & 1586 & 1623 & 52 & 75 & 25 & 27 & 240 & 100 & 1500 & 80 & 17704 & 7976 & 58905 & 2297 & 0.160 & 0.231 & 540 \\
\hline & & & & & & & & & & & & & & & & mean: & 0.261 & 0.336 & 455 \\
\hline & & & & & & & & & & & & & & & prop. 2 & & & & 48 \\
\hline & & & & & & & & & & & & & & & & & & $\mathrm{n}:$ & 12 \\
\hline GR35_spot13 & 1363 & 1572 & 1586 & 1620 & 46 & 54 & 23 & 20 & 915 & 630 & 3100 & 460 & 66115 & 36179 & 75826 & 9784 & 0.295 & 0.436 & 360 \\
\hline GR35_spot19 & 1356 & 1571 & 1583 & 1618 & 40 & 55 & 20 & 16 & 575 & 460 & & 380 & & & & & 0.267 & & 381 \\
\hline GR35_spot14 & 1357 & 1574 & 1583 & 1620 & 39 & 66 & 20 & 22 & 532 & 440 & 1950 & 325 & 32591 & 30883 & 41475 & 7604 & 0.273 & 0.399 & 393 \\
\hline GR35_spot12 & 1356 & 1568 & 1582 & 1618 & 41 & 59 & 20 & 21 & 830 & 520 & 3420 & 480 & 53454 & 32627 & 72742 & 10720 & 0.243 & 0.390 & 394 \\
\hline GR35_spot15 & 1357 & 1573 & 1582 & 1618 & 40 & 59 & 20 & 21 & 815 & 490 & 3700 & 450 & & 30745 & 78697 & 10050 & 0.220 & 0.366 & 413 \\
\hline GR35_spot1 & 1353 & 1540 & 1583 & 1622 & 52 & 80 & 21 & 19 & 133 & 0 & 580 & 49 & 10408 & 0 & 19132 & 990 & 0.229 & 0.341 & 443 \\
\hline GR35_spot17 & 1358 & 1572 & 1583 & 1616 & 41 & 58 & 19 & 18 & 521 & 390 & 1870 & 280 & 22717 & 24056 & 37785 & 5360 & 0.279 & 0.345 & 452 \\
\hline GR35_spot5 & 1355 & 1540 & 1583 & 1622 & 51 & 80 & 22 & 19 & 130 & 0 & & 30 & & 0 & & & 0.206 & 318 & 461 \\
\hline GR35_spot11 & 1357 & 1571 & 1582 & 1616 & 38 & 61 & 22 & 19 & 780 & 500 & 4050 & 340 & 46558 & 32436 & 94755 & 6870 & 0.193 & 0.314 & 461 \\
\hline GR35_spot18 & 1356 & 1572 & 1582 & 1621 & 42 & 55 & 19 & 19 & 915 & 620 & 3910 & 555 & 40869 & 36264 & 79005 & 11214 & 0.234 & 0.312 & 475 \\
\hline GR35_spot20 & 1359 & 1573 & 1583 & 1621 & 39 & 55 & 19 & 14 & 870 & 980 & 3350 & 470 & 36084 & 84666 & 67690 & 10336 & 0.260 & 0.316 & 478 \\
\hline GR35_spot9 & 1354 & 1571 & 1580 & 1618 & 41 & 62 & 23 & 20 & 3750 & 1820 & 15000 & 1820 & 163509 & 120002 & 366898 & 38710 & 0.250 & 0.308 & 484 \\
\hline GR35_spot8 & 1356 & 1572 & 1583 & 1619 & 44 & 65 & 23 & 26 & 1200 & 800 & 5090 & 580 & 56151 & 55301 & 124501 & 16037 & 0.236 & 0.285 & 504 \\
\hline & & & & & & & & & & & & & & & & mean: & 0.245 & 0.349 & 438 \\
\hline & & & & & & & & & & & & & & & $1 \sigma(i$ & nternal) & 0.028 & 0.045 & 44 \\
\hline & & & & & & & & & & & & & & & $1 \mathrm{SE}$ (i & ternal):| & 0.008 & 0.012 & 12 \\
\hline & & & & & & & & & & & & & & & prop. & "is & a & & 37 \\
\hline & & & & & & & & & & & & & & & & & & $\mathrm{n}:$ & 13 \\
\hline GR36_spot12 & 1324 & 1573 & 1605 & 1623 & 79 & 55 & 30 & 15 & 3100 & 230 & 1120 & 070 & 384688 & 13453 & 35733 & 15787 & 2.768 & 0.882 & 67 \\
\hline GR36_spot15 & 1333 & 1573 & 1604 & 1624 & 70 & 63 & 35 & 18 & 7120 & 670 & 2630 & 1400 & & 44889 & & & 2.707 & 0.851 & 107 \\
\hline GR36_spot9 & 1337 & 1571 & 1603 & 1622 & 67 & 52 & 34 & 20 & 6450 & 820 & 2550 & 1040 & 678820 & 66979 & 92203 & 32673 & 2.529 & 0.845 & 131 \\
\hline GR36_spot10 & 1326 & 1573 & 1605 & 1624 & 88 & 55 & 31 & 18 & 3510 & 370 & 1450 & 780 & 328485 & 31966 & 47803 & 22054 & 2.421 & 0.825 & 161 \\
\hline GR36_spot8 & 1334 & 1572 & 1603 & 1623 & 79 & 50 & 33 & 16 & 11300 & 1320 & 4900 & 2650 & 949362 & 103673 & 171963 & 66602 & 2.306 & 0.799 & 196 \\
\hline GR36_spot11 & 1346 & 1573 & 1604 & 1624 & 55 & 81 & 36 & 16 & 15800 & 2600 & 7700 & 2000 & 1365022 & 330810 & 294795 & 34031 & 2.052 & 0.806 & 196 \\
\hline GR36_spot14 & 1346 & 1573 & 1603 & 1622 & 58 & 68 & 34 & 18 & 10500 & 2100 & 4710 & 2400 & 956615 & 224310 & 170304 & 67858 & 2.229 & 0.801 & 197 \\
\hline
\end{tabular}




\begin{tabular}{|c|c|c|c|c|c|c|c|c|c|c|c|c|c|c|c|c|c|c|c|}
\hline GR36_spot7 & 1345 & 1573 & 1603 & 1623 & 52 & 48 & 33 & 16 & 5300 & 590 & 3158 & 1350 & | 432911 & 44485 & 110829 & 22971 & 1.678 & 0.764 & 234 \\
\hline GR36_spot3 & 1343 & 1550 & 1603 & 1622 & 77 & 70 & 46 & 20 & 660 & 40 & 420 & 80 & 79828 & 2978 & 30348 & 1702 & 1.571 & 0.714 & 281 \\
\hline GR36_spot1 & 1343 & 1540 & 1602 & 1622 & 77 & 80 & 39 & 19 & 740 & 50 & 550 & 180 & 83723 & 4254 & 33694 & 3637 & 1.345 & 0.692 & 285 \\
\hline GR36_spot6 & 1346 & 1540 & 1603 & 1620 & 64 & 80 & 51 & 20 & 1350 & 90 & 780 & 60 & 131334 & 7657 & 50377 & 1276 & 1.731 & 0.718 & 285 \\
\hline GR36_spot4 & 1346 & 1550 & 1604 & 1626 & 69 & 70 & 44 & 21 & 1500 & 150 & 1030 & 190 & 162577 & 11166 & 71188 & 4243 & 1.456 & 0.683 & 305 \\
\hline GR36_spot2 & 1346 & 1550 & 1605 & 1623 & 72 & 70 & 46 & 20 & 660 & 35 & 440 & 90 & 72233 & 2606 & 31793 & 1914 & 1.500 & 0.682 & 310 \\
\hline \multirow[t]{6}{*}{ GR36_spot5 } & \multirow[t]{6}{*}{1348} & \multirow[t]{6}{*}{1545} & \multirow[t]{6}{*}{1603} & \multirow[t]{6}{*}{1622} & \multirow[t]{6}{*}{65} & \multirow[t]{6}{*}{80} & 46 & 25 & 1280 & 100 & 860 & 210 & 130690 & 8508 & 62141 & 5583 & 1.488 & 0.659 & 333 \\
\hline & & & & & & & & & & & & & & & & mean: & 1.984 & 0.766 & 221 \\
\hline & & & & & & & & & & & & & & & $1 \sigma(\mathrm{i}$ & nternal): & 0.485 & 0.071 & 80 \\
\hline & & & & & & & & & & & & & & & 1 SE (i & ternal):| & 0.130 & 0.019 & 21 \\
\hline & & & & & & & & & & & & & & & prop. $2 \mathrm{~S}$ & E (interna & and & ternal): & 50 \\
\hline & & & & & & & & & & & & & & & & & & $\mathrm{n}:$ & 14 \\
\hline GR38_spot12 & 1346 & 1577 & 1606 & 1623 & 60 & 65 & 36 & 29 & 3150 & 540 & 1780 & 310 & 296881 & 55135 & 68147 & 9561 & 1.770 & 0.813 & 185 \\
\hline GR38_spot13 & 1345 & 1577 & 1606 & 1624 & 61 & 71 & 37 & 27 & 4450 & 800 & 2500 & 360 & 426393 & 89221 & 98371 & 10337 & 1.780 & 0.797 & 203 \\
\hline GR38_spot11 & 1345 & 1576 & 1606 & 1621 & 62 & 75 & 39 & 28 & 6800 & 1000 & 4350 & 250 & 662248 & 117810 & 180418 & 7444 & 1.563 & 0.786 & 203 \\
\hline GR38_spot10 & 1343 & 1577 & 1605 & 1623 & 62 & 83 & 37 & 26 & 12700 & 2600 & 8200 & 900 & 1236845 & 338978 & 322657 & 24885 & 1.549 & 0.781 & 208 \\
\hline GR38_spot17 & 1343 & 1576 & 1602 & 1624 & 60 & 67 & 39 & 19 & 8760 & 1730 & 4700 & 930 & 825611 & 182071 & 194934 & 18792 & 1.864 & 0.794 & 208 \\
\hline GR38_spot15 & 1344 & 1577 & 1606 & 1626 & 61 & 76 & 40 & 18 & 5650 & 830 & 3400 & 250 & 541375 & 99086 & 144632 & 4786 & 1.662 & 0.784 & 211 \\
\hline GR38_spot9 & 1344 & 1575 & 1604 & 1623 & 63 & 85 & 41 & 26 & 4500 & 1050 & 2600 & 250 & 445321 & 140194 & 113366 & 6913 & 1.731 & 0.787 & 211 \\
\hline GR38_spot16 & 1346 & 1577 & 1604 & 1625 & 62 & 73 & 39 & 21 & 10350 & 1980 & 6300 & 850 & 1007980 & 227043 & 261295 & 18983 & 1.643 & 0.782 & 212 \\
\hline GR38_spot14 & 1344 & 1576 & 1604 & 1628 & 60 & 65 & 36 & 21 & 11400 & 1850 & 8100 & 1700 & 1074425 & 188888 & 310109 & 37966 & 1.407 & 0.755 & 224 \\
\hline GR38_spot8 & 1345 & 1577 & 1605 & 1622 & 55 & 75 & 41 & 30 & 11350 & 2050 & 6650 & 300 & 980570 & 241510 & 289956 & 9571 & 1.707 & 0.766 & 233 \\
\hline GR38_spot7 & 1347 & 1545 & 1608 & 1620 & 69 & 80 & 54 & 25 & 540 & 55 & 350 & 10 & 58528 & 4679 & 20100 & 266 & 1.543 & 0.744 & 247 \\
\hline GR38_spot2 & 1347 & 1545 & 1603 & 1620 & 67 & 80 & 51 & 20 & 300 & 35 & 185 & 25 & 30553 & 2978 & 10034 & 532 & 1.622 & 0.743 & 253 \\
\hline GR38_spot4 & 1342 & 1545 & 1605 & 1623 & 71 & 80 & 38 & 20 & 150 & 0 & 90 & 33 & 16729 & 0 & 5372 & 702 & 1.667 & 0.734 & 265 \\
\hline GR38_spot5 & 1348 & 1545 & 1604 & 1620 & 62 & 80 & 50 & 25 & 775 & 40 & 460 & 60 & 75477 & 3403 & 27960 & 1595 & 1.685 & 0.719 & 282 \\
\hline & & & & & & & & & & & & & & & & mean: & 1.657 & 0.770 & 225 \\
\hline & & & & & & & & & & & & & & & $1 \sigma(i$ & nternal): & 0.112 & 0.026 & 26 \\
\hline & & & & & & & & & & & & & & & $1 \mathrm{SE}$ (i & nternal): | & 0.030 & 0.007 & 7 \\
\hline & & & & & & & & & & & & & & & prop. $2 \mathrm{~S}$ & E (interna & land & ternal): & 30 \\
\hline & & & & & & & & & & & & & & & & & & $\mathrm{n}:$ & 14 \\
\hline GR49_spot17 & 1352 & 1565 & 1581 & 1622 & 48 & 85 & 20 & 16 & 1350 & 40 & 3300 & 290 & 68913 & 5341 & 103673 & 4935 & 0.409 & 0.388 & 441 \\
\hline GR49_spot3 & 1353 & 1540 & 1581 & 1623 & 48 & 80 & 20 & 17 & 85 & 5 & 360 & 12 & 5995 & 425 & 11310 & 217 & 0.236 & 0.342 & 444 \\
\hline GR49_spot11 & 1355 & 1568 & 1582 & 1624 & 47 & 75 & 21 & 9 & 330 & 50 & 1470 & 100 & 24363 & 5890 & 48490 & 957 & 0.224 & 0.330 & 453 \\
\hline GR49_spot4 & 1351 & 1540 & 1581 & 1622 & 53 & 80 & 21 & 25 & 91 & 5 & 390 & 35 & 6353 & 425 & 12865 & 931 & 0.233 & 0.315 & 472 \\
\hline GR49_spot6 & 1350 & 1540 & 1580 & 1622 & 52 & 80 & 21 & 20 & 125 & 0 & 560 & 28 & 8561 & 0 & 18473 & 596 & 0.223 & 0.310 & 474 \\
\hline GR49_spot13 & 1355 & 1570 & 1581 & 1623 & 44 & 70 & 19 & 7 & 730 & 15 & 3300 & 150 & 34159 & 1649 & 98489 & 1117 & 0.221 & 0.255 & 532 \\
\hline GR49_spot10 & 1354 & 1570 & 1582 & 1623 & 43 & 70 & 18 & 10 & 430 & 15 & 2175 & 60 & 19664 & 1649 & 61497 & 638 & 0.198 & 0.242 & 539 \\
\hline GR49_spot15 & 1359 & 1570 & 1581 & 1624 & 42 & 75 & 18 & 10 & 500 & 24 & 2550 & 90 & 22333 & 2827 & 72100 & 957 & 0.196 & 0.234 & 547 \\
\hline GR49_spot9 & 1357 & 1570 & 1581 & 1622 & 42 & 70 & 19 & 19 & 810 & 50 & 3700 & 220 & 36179 & 5498 & 110427 & 4445 & 0.219 & 0.240 & 548 \\
\hline GR49_spot12 & 1354 & 1570 & 1581 & 1621 & 48 & 70 & 19 & 14 & 360 & 10 & 2290 & 120 & 18377 & 1100 & 68345 & 1787 & 0.157 & 0.208 & 564 \\
\hline GR49_spot18 & 1355 & 1560 & 1581 & 1620 & 43 & 75 & 20 & 14 & 670 & 60 & 3670 & 240 & 30639 & 7069 & 115296 & 3573 & 0.183 & 0.205 & 575 \\
\hline GR49_spot8 & 1356 & 1571 & 1582 & 1622 & 40 & 70 & 19 & 10 & 610 & 20 & 3540 & 40 & 25949 & 2199 & 105652 & 425 & 0.172 & 0.197 & 580 \\
\hline GR49_spot14 & 1357 & 1570 & 1582 & 1623 & 38 & 70 & 18 & 22 & 630 & 20 & 4150 & 290 & 25460 & 2199 & 117338 & 6785 & 0.152 & 0.170 & 603 \\
\hline GR49_spot16 & 1357 & 1560 & 1582 & 1620 & 40 & 80 & 18 & 11 & 465 & 90 & 3750 & 110 & 19781 & 11310 & 106029 & 1287 & 0.124 & 0.156 & 609 \\
\hline & & & & & & & & & & & & & & & & mean: & 0.211 & 0.257 & 527 \\
\hline & & & & & & & & & & & & & & & $1 \sigma(\mathrm{i}$ & nternal): & 0.064 & 0.067 & 57 \\
\hline & & & & & & & & & & & & & & & 1 SE (i & nternal):| & 0.017 & 0.018 & 15 \\
\hline & & & & & & & & & & & & & & & prop. $2 \mathrm{~S}$ & E (interna & I and $\mathrm{e}$ & ternal): & 41 \\
\hline & & & & & & & & & & & & & & & & & & $\mathrm{n}:$ & 14 \\
\hline GR50_spot8 & 1351 & 1535 & 1583 & 1610 & 98 & 80 & 24 & 60 & 1450 & 340 & 4180 & 950 & 223210 & 28926 & 157582 & 60618 & 0.347 & 0.506 & 299 \\
\hline GR50_spot21 & 1350 & 1550 & 1584 & 1615 & 87 & 80 & 19 & 50 & 655 & 170 & 2650 & 470 & 89512 & 14463 & 79090 & 24992 & 0.247 & 0.462 & 319 \\
\hline GR50_spot9 & 1349 & 1545 & 1583 & 1617 & 95 & 80 & 20 & 40 & 650 & 200 & 3310 & 450 & 96997 & 17016 & 103987 & 19143 & 0.196 & 0.441 & 327 \\
\hline GR50_spot7 & 1350 & 1540 & 1584 & 1615 & 73 & 80 & 25 & 60 & 1000 & 300 & 2600 & 480 & 114668 & 25523 & 102102 & 30628 & 0.385 & 0.463 & 355 \\
\hline GR50_spot18 & 1350 & 1540 & 1584 & 1622 & 75 & 75 & 18 & 35 & 360 & 50 & 3440 & 180 & 42412 & 3988 & 97264 & 6700 & 0.105 & 0.290 & 461 \\
\hline GR50_spot10 & 1356 & 1550 & 1584 & 1625 & 58 & 70 & 20 & 15 & 127 & 10 & 1020 & 25 & 11570 & 744 & 32044 & 399 & 0.125 & 0.263 & 496 \\
\hline GR50_spot12 & 1355 & 1545 & 1584 & 1623 & 60 & 75 & 21 & 24 & 110 & 20 & 925 & 60 & 10032 & 1595 & 30513 & 1531 & 0.119 & 0.238 & 520 \\
\hline GR50_spot16 & 1355 & 1540 & 1584 & 1624 & 57 & 75 & 19 & 20 & 240 & 60 & 2300 & 120 & 21488 & 4786 & 68644 & 2552 & 0.104 & 0.232 & 522 \\
\hline GR50_spot19 & 1356 & 1540 & 1584 & 1625 & 48 & 75 & 18 & 20 & 210 & 10 & 1600 & 80 & 13788 & 798 & 45239 & 1702 & 0.131 & 0.227 & 536 \\
\hline GR50_spot11 & 1354 & 1550 & 1584 & 1626 & 48 & 70 & 20 & 15 & 230 & 20 & 1680 & 60 & 15661 & 1489 & 52779 & 957 & 0.137 & 0.226 & 539 \\
\hline & & & & & & & & & & & & & & & & mean: & 0.190 & 0.335 & 437 \\
\hline & & & & & & & & & & & & & & & $1 \sigma(i$ & nternal): & 0.098 & 0.111 & 95 \\
\hline & & & & & & & & & & & & & & & 1 SE (i & nternal): & 0.031 & 0.035 & 30 \\
\hline & & & & & & & & & & & & & & & prop. 2 & E linto & and & & 68 \\
\hline
\end{tabular}




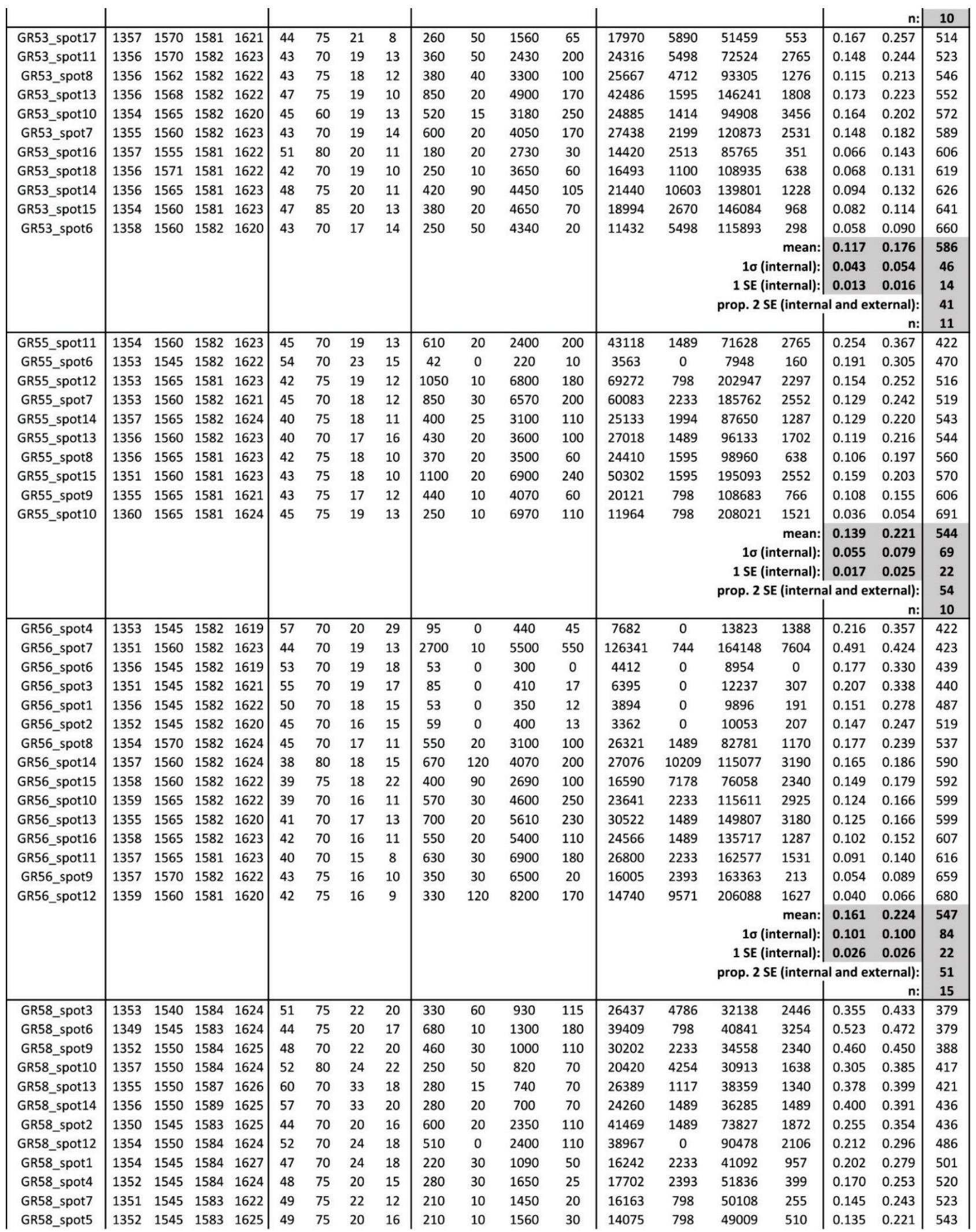




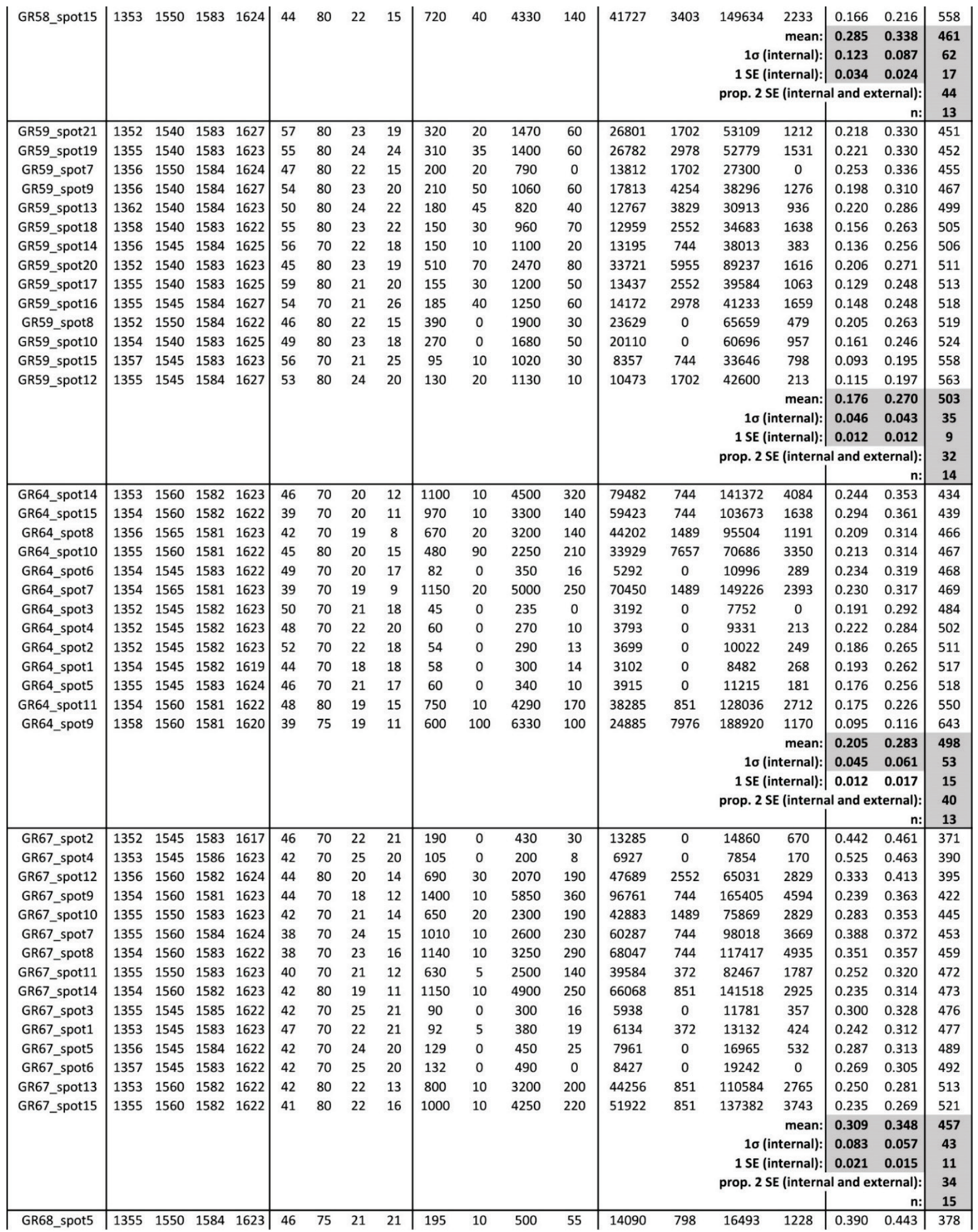




\begin{tabular}{|c|c|c|c|c|c|c|c|c|c|c|c|c|c|c|c|c|c|c|c|}
\hline GR68_spot4 & 1354 & 1545 & 1582 & 1620 & 48 & 70 & 20 & 22 & 190 & 0 & 720 & 55 & 14326 & 0 & 22619 & 1287 & 0.264 & 0.375 & 416 \\
\hline GR68_spot6 & 1354 & 1550 & 1583 & 1622 & 41 & 75 & 24 & 21 & 185 & 0 & 500 & 40 & 11914 & 0 & 18850 & 893 & 0.370 & 0.376 & 444 \\
\hline GR68_spot14 & 1355 & 1560 & 1582 & 1623 & 46 & 80 & 24 & 20 & 1150 & 40 & 3300 & 320 & 72360 & 3403 & 124407 & 6806 & 0.348 & 0.355 & 460 \\
\hline GR68_spot11 & 1355 & 1565 & 1582 & 1622 & 47 & 70 & 24 & 10 & 1470 & 10 & 4780 & 230 & 94506 & 744 & 180202 & 3613 & 0.308 & 0.340 & 466 \\
\hline GR68_spot10 & 1354 & 1565 & 1582 & 1623 & 48 & 70 & 25 & 16 & 2000 & 240 & 7010 & 580 & 141056 & 17866 & 275282 & 14577 & 0.285 & 0.327 & 473 \\
\hline GR68_spot12 & 1354 & 1565 & 1582 & 1622 & 45 & 70 & 25 & 17 & 1590 & 10 & 6100 & 350 & 101501 & 744 & 231810 & 9346 & 0.261 & 0.305 & 490 \\
\hline GR68_spot8 & 1354 & 1565 & 1582 & 1621 & 47 & 90 & 26 & 25 & 2050 & 500 & 5400 & 820 & 126906 & 47856 & 220540 & 32201 & 0.380 & 0.334 & 491 \\
\hline GR68_spot13 & 1354 & 1565 & 1582 & 1622 & 44 & 70 & 23 & 16 & 1450 & 10 & 5900 & 400 & 90507 & 744 & 206273 & 8430 & 0.246 & 0.297 & 494 \\
\hline GR68_spot7 & 1355 & 1565 & 1583 & 1623 & 42 & 90 & 26 & 13 & 990 & 60 & 2700 & 190 & 54766 & 5743 & 110270 & 2627 & 0.367 & 0.327 & 495 \\
\hline GR68_spot16 & 1355 & 1565 & 1582 & 1625 & 43 & 75 & 24 & 21 & 1050 & 30 & 4000 & 170 & 61759 & 2393 & 145926 & 3797 & 0.263 & 0.292 & 505 \\
\hline GR68_spot15 & 1356 & 1565 & 1584 & 1624 & 45 & 70 & 24 & 18 & 900 & 10 & 3000 & 190 & 49235 & 744 & 113097 & 3637 & 0.300 & 0.297 & 509 \\
\hline \multirow[t]{6}{*}{ GR68_spot9 } & \multirow[t]{6}{*}{1359} & \multirow[t]{6}{*}{1560} & \multirow[t]{6}{*}{1581} & \multirow[t]{6}{*}{1623} & \multirow[t]{6}{*}{47} & \multirow[t]{6}{*}{80} & \multirow[t]{6}{*}{22} & \multirow[t]{6}{*}{15} & \multirow[t]{6}{*}{1280} & \multirow[t]{6}{*}{110} & \multirow[t]{6}{*}{8330} & \multirow[t]{6}{*}{200} & \multirow[t]{6}{*}{82291} & \multirow[t]{6}{*}{9359} & 287864 & 4712 & 0.154 & 0.220 & \multirow{6}{*}{$\begin{array}{c}550 \\
475 \\
42 \\
12 \\
36 \\
13 \\
\end{array}$} \\
\hline & & & & & & & & & & & & & & & & mea & 0.303 & 0.330 & \\
\hline & & & & & & & & & & & & & & & $1 \sigma($ & iterna & 0.065 & 0.051 & \\
\hline & & & & & & & & & & & & & & & $1 \mathrm{SE}$ & ren & 0.018 & 0.014 & \\
\hline & & & & & & & & & & & & & & & \multirow{2}{*}{\multicolumn{4}{|c|}{ prop. 2 SE (internal and external): }} & \\
\hline & & & & & & & & & & & & & & & & & & & \\
\hline GR69_spot6 & 1353 & 1550 & 1584 & 1625 & 39 & 80 & 22 & 16 & 330 & 0 & 570 & 60 & 20216 & 0 & 19698 & 1021 & 0.579 & 0.494 & 369 \\
\hline GR69_spot3 & 1353 & 1550 & 1584 & 1623 & 38 & 75 & 20 & 18 & 470 & 0 & 940 & 140 & 28054 & 0 & 29531 & 2680 & 0.500 & 0.466 & 380 \\
\hline GR69_spot4 & 1352 & 1550 & 1584 & 1624 & 38 & 75 & 21 & 11 & 360 & 0 & 700 & 90 & 20794 & 0 & 23091 & 1053 & 0.514 & 0.463 & 387 \\
\hline GR69_spot12 & 1351 & 1565 & 1582 & 1617 & 51 & 60 & 24 & 35 & 900 & 120 & 3070 & 340 & 67442 & 7657 & 100784 & 12655 & 0.293 & 0.401 & 397 \\
\hline GR69_spot16 & 1354 & 1565 & 1583 & 1623 & 37 & 75 & 21 & 15 & 2650 & 40 & 5000 & 670 & 144068 & 3190 & 164934 & 10688 & 0.530 & 0.451 & 404 \\
\hline GR69_spot13 & 1357 & 1560 & 1583 & 1622 & 39 & 70 & 24 & 18 & 3500 & 280 & 5650 & 850 & 200564 & 20844 & 213000 & 16271 & 0.619 & 0.467 & 407 \\
\hline GR69_spot14 & 1354 & 1560 & 1583 & 1622 & 35 & 70 & 21 & 16 & 3200 & 10 & 5950 & 920 & 170247 & 744 & 196271 & 15654 & 0.538 & 0.445 & 412 \\
\hline GR69_spot5 & 1352 & 1550 & 1584 & 1624 & 41 & 80 & 26 & 22 & 298 & 10 & 550 & 85 & 19192 & 851 & 22462 & 1989 & 0.542 & 0.440 & 418 \\
\hline GR69_spot17 & 1354 & 1565 & 1583 & 1623 & 36 & 75 & 22 & 15 & 2550 & 10 & 5190 & 650 & 134885 & 798 & 173561 & 10369 & 0.491 & 0.423 & 424 \\
\hline GR69_spot8 & 1355 & 1570 & 1583 & 1622 & 39 & 70 & 22 & 16 & 1250 & 20 & 3500 & 320 & 74103 & 1489 & 113139 & 5445 & 0.357 & 0.385 & 431 \\
\hline GR69_spot15 & 1354 & 1565 & 1583 & 1622 & 36 & 75 & 21 & 14 & 2400 & 100 & 5000 & 600 & 118184 & 7976 & 164934 & 8933 & 0.480 & 0.405 & 441 \\
\hline GR69_spot11 & 1354 & 1570 & 1583 & 1621 & 36 & 70 & 24 & 21 & 2750 & 10 & 6500 & 650 & 135419 & 744 & 213387 & 14516 & 0.423 & 0.373 & 461 \\
\hline GR69_spot10 & 1352 & 1570 & 1583 & 1621 & 29 & 70 & 24 & 17 & 3050 & 20 & 5850 & 700 & 134450 & 1489 & 199171 & 12655 & 0.521 & 0.388 & 469 \\
\hline \multirow[t]{6}{*}{ GR69_spot9 } & \multirow[t]{6}{*}{1351} & \multirow[t]{6}{*}{1570} & \multirow[t]{6}{*}{1581} & 1620 & 48 & 70 & 19 & 32 & 750 & 50 & 8100 & 150 & 56549 & 3722 & 233938 & 5105 & 0.093 & 0.195 & 558 \\
\hline & & & & & & & & & & & & & & & & mean: & 0.463 & 0.414 & 425 \\
\hline & & & & & & & & & & & & & & & $10(i$ & iternal): & 0.131 & 0.070 & 46 \\
\hline & & & & & & & & & & & & & & & $1 \mathrm{SE}$ ( & iternal) & 0.035 & 0.019 & 12 \\
\hline & & & & & & & & & & & & & & & prop. $2 \mathrm{~s}$ & (inte & I and & rnal): & 36 \\
\hline & & & & & & & & & & & & & & & & & & $\mathrm{n}:$ & 14 \\
\hline
\end{tabular}

Discussion DR2: Supporting data for vitrinite reflectance thermometry

Multiple random reflectance measurements on solid bitumen $\left(\mathrm{R}_{\mathrm{SB}}\right)$ were made from each sample, as no primary vitrinite fragments were identified. Data from individual analyses from each sample are shown on Table DR2. These $\mathrm{R}_{\mathrm{SB}}$ values were converted into equivalent vitrinite reflectance $\left(R_{V E}\right)$ values using the equation of Jacob (1989) $\left(R_{V E}=0.618 * R_{S B}+0.40\right)$. The mean and 1 standard deviation for all $\mathrm{R}_{\mathrm{SB}}$ and $\mathrm{R}_{\mathrm{VE}}$ data from each sample are shown at the bottom of Table DR2. Peak temperatures and uncertainties are shown on Table 2 in the text, and were obtained from the mean $\mathrm{R}_{\mathrm{VE}}$ value plus or minus 2 standard deviations, using the equation for burial heating from Barker and Pawlewicz (1994): $\left(T_{\text {peak }}=\left(\ln \left(R_{V E}\right)+1.68\right) / 0.0124\right)$. 
Table DR2: Supporting data for peak temperature determinations from vitrinite reflectance.

\begin{tabular}{|c|c|c|c|c|c|c|c|c|c|c|c|c|}
\hline \multirow[t]{2}{*}{ sample } & \multicolumn{2}{|c|}{ GR04 } & \multicolumn{2}{|c|}{ GR08B } & \multicolumn{2}{|c|}{ GR09 } & \multicolumn{2}{|c|}{ GR14 } & \multicolumn{2}{|c|}{ GR37 } & \multicolumn{2}{|c|}{ GR42 } \\
\hline & $\mathbf{R}_{\mathrm{SB}}(\%)$ & $\mathbf{R}_{\mathrm{VE}}(\%)$ & $\mathbf{R}_{\mathrm{SB}}(\%)$ & $\mathbf{R}_{\mathrm{VE}}(\%)$ & $\mathrm{R}_{\mathrm{SB}}(\%)$ & $\mathbf{R}_{\mathrm{VE}}(\%)$ & $\mathbf{R}_{\mathrm{SB}}(\%)$ & $\mathbf{R}_{\mathrm{VE}}(\%)$ & $\mathbf{R}_{\mathrm{SB}}(\%)$ & $\mathbf{R}_{\mathrm{VE}}(\%)$ & $\mathbf{R}_{\mathrm{SB}}(\%)$ & $\mathbf{R}_{\mathrm{VE}}(\%)$ \\
\hline random & 0.92 & 0.97 & 0.91 & 0.96 & 0.85 & 0.93 & 0.77 & 0.88 & 0.75 & 0.86 & 0.71 & 0.84 \\
\hline reflectance & 1.06 & 1.06 & 1.43 & 1.28 & 0.91 & 0.96 & 0.8 & 0.89 & 0.75 & 0.86 & 0.75 & 0.86 \\
\hline values & 1.07 & 1.06 & & & 1.01 & 1.02 & 0.83 & 0.91 & 0.79 & 0.89 & 0.77 & 0.88 \\
\hline & 1.11 & 1.09 & & & 1.04 & 1.04 & 0.83 & 0.91 & 0.8 & 0.89 & 0.79 & 0.89 \\
\hline & 1.11 & 1.09 & & & 1.06 & 1.06 & 0.84 & 0.92 & 0.92 & 0.97 & 0.82 & 0.91 \\
\hline & 1.15 & 1.11 & & & 1.28 & 1.19 & 0.84 & 0.92 & 1.15 & 1.11 & 0.82 & 0.91 \\
\hline & 1.16 & 1.12 & & & 1.44 & 1.29 & 0.85 & 0.93 & 1.22 & 1.15 & 0.83 & 0.91 \\
\hline & 1.18 & 1.13 & & & 1.55 & 1.36 & 0.86 & 0.93 & & & 0.84 & 0.92 \\
\hline & 1.18 & 1.13 & & & & & 0.93 & 0.97 & & & 0.88 & 0.94 \\
\hline & 1.19 & 1.14 & & & & & 0.96 & 0.99 & & & 0.88 & 0.94 \\
\hline & 1.2 & 1.14 & & & & & 0.96 & 0.99 & & & 0.89 & 0.95 \\
\hline & 1.22 & 1.15 & & & & & 1.02 & 1.03 & & & 0.89 & 0.95 \\
\hline & 1.24 & 1.17 & & & & & 1.12 & 1.09 & & & 0.91 & 0.96 \\
\hline & 1.24 & 1.17 & & & & & 1.16 & 1.12 & & & 0.92 & 0.97 \\
\hline & 1.25 & 1.17 & & & & & 1.21 & 1.15 & & & 0.94 & 0.98 \\
\hline & 1.26 & 1.18 & & & & & 1.21 & 1.15 & & & 0.95 & 0.99 \\
\hline & 1.29 & 1.20 & & & & & & & & & 0.96 & 0.99 \\
\hline & 1.29 & 1.20 & & & & & & & & & 1.02 & 1.03 \\
\hline & 1.31 & 1.21 & & & & & & & & & 1.03 & 1.04 \\
\hline & 1.31 & 1.21 & & & & & & & & & 1.04 & 1.04 \\
\hline & 1.32 & 1.22 & & & & & & & & & 1.06 & 1.06 \\
\hline & 1.32 & 1.22 & & & & & & & & & 1.12 & 1.09 \\
\hline & 1.36 & 1.24 & & & & & & & & & 1.15 & 1.11 \\
\hline & 1.37 & 1.25 & & & & & & & & & & \\
\hline & 1.38 & 1.25 & & & & & & & & & & \\
\hline & 1.41 & 1.27 & & & & & & & & & & \\
\hline & 1.42 & 1.28 & & & & & & & & & & \\
\hline & 1.45 & 1.30 & & & & & & & & & & \\
\hline & 1.46 & 1.30 & & & & & & & & & & \\
\hline & 1.54 & 1.35 & & & & & & & & & & \\
\hline mean & 1.26 & 1.18 & 1.17 & 1.12 & 1.14 & 1.11 & 0.95 & 0.99 & 0.91 & 0.96 & 0.91 & 0.96 \\
\hline $1 \sigma$ & 0.136 & 0.083 & 0.368 & 0.161 & 0.253 & 0.146 & 0.150 & 0.090 & 0.196 & 0.112 & 0.117 & 0.071 \\
\hline $\mathrm{n}$ & 30 & 30 & 2 & 2 & 8 & 8 & 16 & 16 & 7 & 7 & 23 & 23 \\
\hline
\end{tabular}

Discussion DR3: Supporting data for Rock-Eval pyrolysis thermometry

Supporting data for Rock-Eval pyrolysis analyses, including measured parameters such as the $\mathrm{S} 1, \mathrm{~S} 2$, and $\mathrm{S} 3$ peaks, and $\mathrm{T}_{\max }$, are shown in Table DR3. $\mathrm{T}_{\max }$ was converted into cal. $\mathrm{R}_{\mathrm{V}}$ using the equation: cal. $\mathrm{R}_{\mathrm{V}}=0.0180 * \mathrm{~T}_{\max }-7.16$ (Jarvie et al., 2001), and $\mathrm{T}_{\text {peak }}$ was calculated 
from cal. $R_{V}$ using the equation: $T_{\text {peak }}=\left(\ln \left(\right.\right.$ cal. $\left.\left.R_{V}\right)+1.68\right) / 0.0124$ (Barker and Pawlewicz, 1994). Errors of \pm 0.2 cal. $R_{V}$ were assigned to $T_{\text {peak }}$ estimates, which results in a typical error range of $\pm 15-30^{\circ} \mathrm{C}$, which is comparable to the typical $\pm 2 \sigma$ error range for vitrinite reflectance peak temperature estimates.

Table DR3: Supporting data for peak temperature determinations from Rock-Eval pyrolysis.

\begin{tabular}{|c|c|c|c|c|c|c|c|c|c|c|c|c|c|c|}
\hline sample & TOC & S1 & S2 & S3 & $\begin{array}{l}\mathrm{T}_{\max } \\
\left({ }^{\circ} \mathrm{C}\right) \\
\end{array}$ & $\mathrm{HI}$ & OI & S2/S3 & $\begin{array}{l}\text { (S1/TOC) } \\
{ }^{* 100}\end{array}$ & PI & $\begin{array}{c}\text { cal. } \\
\mathrm{R}_{\mathrm{v}}(\%)\end{array}$ & $\begin{array}{l}T_{\text {peak }} \\
\left({ }^{\circ} \mathrm{C}\right) \\
\end{array}$ & $\begin{array}{l}-0.2 \text { cal. } R_{v} \\
\text { error }\left({ }^{\circ} \mathrm{C}\right)\end{array}$ & $\begin{array}{l}+0.2 \text { cal. } R_{V} \\
\text { error }\left({ }^{\circ} \mathrm{C}\right)\end{array}$ \\
\hline GR08B & 0.72 & 0.21 & 1.40 & 0.30 & 447 & 194 & 42 & 4.7 & 30 & 0.13 & 0.89 & 126 & 21 & 16 \\
\hline GR09 & 0.08 & 0.07 & 0.40 & 0.19 & 433 & 506 & 241 & 2.1 & 88 & 0.15 & 0.63 & 99 & 31 & 22 \\
\hline GR14 & 2.18 & 0.17 & 1.54 & 0.98 & 440 & 71 & 45 & 1.6 & 8 & 0.10 & 0.76 & 113 & 25 & 19 \\
\hline \multicolumn{15}{|c|}{ Abbreviations: } \\
\hline \multicolumn{15}{|c|}{ TOC: total organic carbon (weight \%) } \\
\hline \multicolumn{15}{|c|}{ S1: volatile hydrocarbon $(\mathrm{HC}$ ) content (mg HC / g rock) } \\
\hline \multicolumn{15}{|c|}{ S2: remaining $\mathrm{HC}$ generative potential (mg HC/g rock) } \\
\hline \multicolumn{15}{|c|}{ S3: carbon dioxide content ( $\mathrm{mg} \mathrm{CO} / 2 / \mathrm{g}$ rock) } \\
\hline \multicolumn{15}{|c|}{$\mathrm{T}_{\max }$ : oven temperature at which maximum amount of $\mathrm{S} 2$ hydrocarbons are released $\left({ }^{\circ} \mathrm{C}\right)$} \\
\hline \multicolumn{15}{|c|}{ HI: hydrogen index $=(\mathrm{S} 2 \times 100) / \mathrm{TOC}(\mathrm{mg} \mathrm{HC} / \mathrm{g}$ TOC $)$} \\
\hline \multicolumn{15}{|c|}{ OI: oxygen index $=(\mathrm{S} 3 \times 100) / \mathrm{TOC}(\mathrm{mg} \mathrm{CO} / 2 / \mathrm{g}$ TOC $)$} \\
\hline \multicolumn{15}{|c|}{$\mathrm{PI}:$ production index $=\mathrm{S} 1 /(\mathrm{S} 1+\mathrm{S} 2)$} \\
\hline \multicolumn{15}{|c|}{ Cal. $R_{V}=$ calculated vitrinite reflectance, calculated from Jarvie et al. (2001): cal. $R_{V}=0.0180 * T_{\max }-7.16$} \\
\hline \multicolumn{15}{|c|}{$T_{\text {peak }}=$ peak temperature; calculated from Barker and Pawlewicz (1994): $T_{\text {peak }}=\left(\ln \left(\right.\right.$ cal. $\left.\left.R_{v}\right)+1.68\right) / 0.0124$} \\
\hline
\end{tabular}


Figure DR1: Pyrograms for Rock-Eval pyrolysis analysis of each sample (Abbreviation: FID = flame ionization detector).
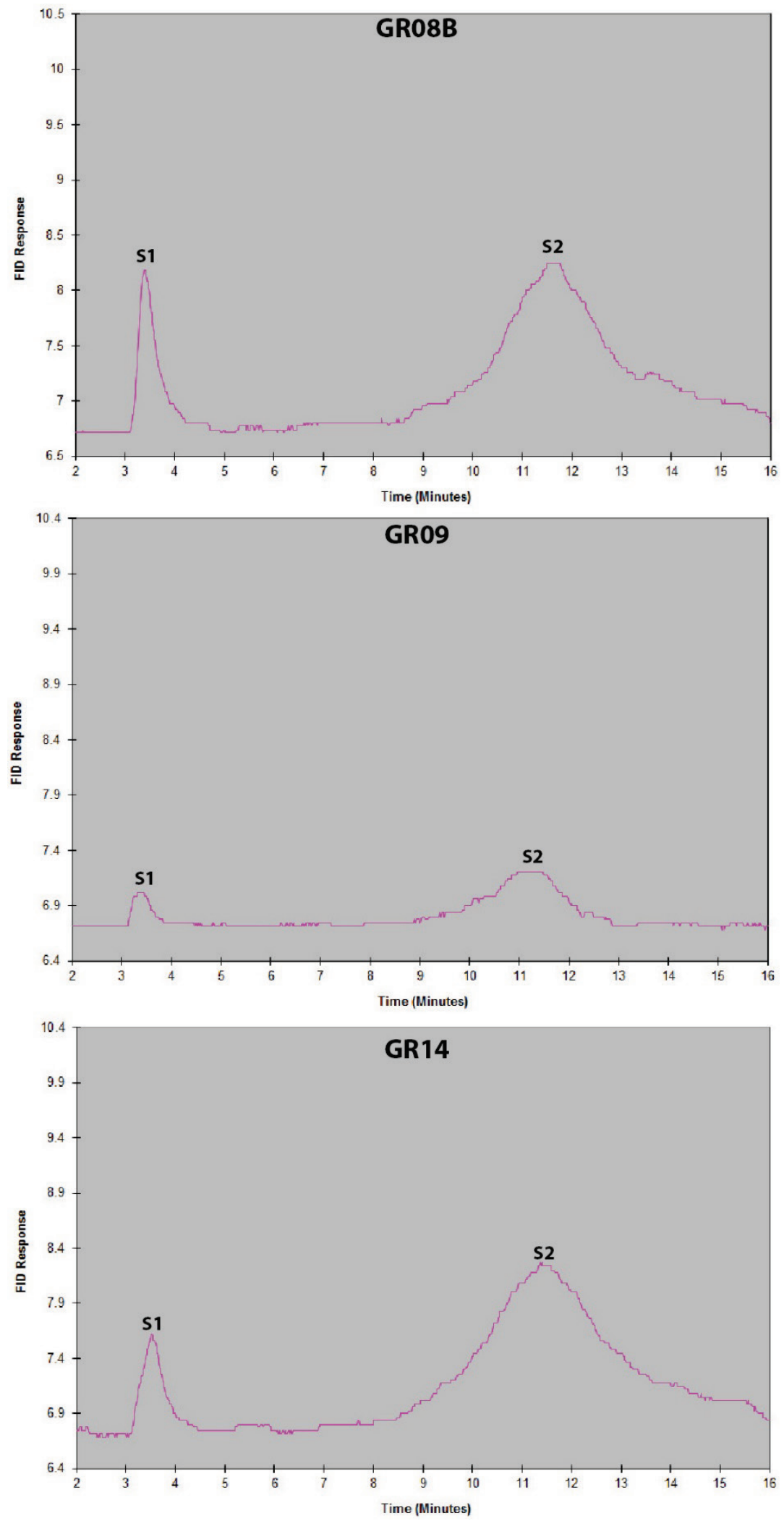


\section{References cited}

Barker, C.E., and Pawlewicz, M.J., 1994, Calculation of vitrinite reflectance from thermal histories and peak temperature: A comparison of methods, in Mukhopadhyay, P.K., and Dow, W.G., eds., Vitrinite Reflectance as a Maturity Parameter: Applications and Limitations: Washington D.C., American Chemical Society, Symposium Series v. 570, p. 216-229.

Cooper, F.J., Hodges, K.V, and Adams, B.A., 2013, Metamorphic constraints on the character and displacement of the South Tibetan fault system, central Bhutanese Himalaya: Lithosphere, $v$. 5, p. 67-81, doi: 10.1130/L221.1.

Jacob, H., 1989, Classification, structure, genesis and practical importance of natural solid oil bitumen ("migrabitumen"): International Journal of Coal Geology, v. 11, p. 65-79, doi: 10.1016/0166-5162(89)90113-4.

Jarvie, D.M., Claxton, B.L., Henk, F., and Breyer, J.T., 2001, Oil and shale gas from the Barnett Shale, Ft. Worth Basin, Texas: American Association of Petroleum Geologists Bulletin, v. 85 (supplement), p. A100.

Rahl, J.M., Anderson, K.M., Brandon, M.T., and Fassoulas, C., 2005, Raman spectroscopic carbonaceous material thermometry of low-grade metamorphic rocks: Calibration and application to tectonic exhumation in Crete, Greece: Earth and Planetary Science Letters, v. 240, p. 339-354, doi: 10.1016/j.eps1.2005.09.055. 